\title{
Effectiveness of systematic treatment selection for psychodynamic and cognitive-behavioural therapy: randomised controlled trial in routine mental healthcare ${ }^{\dagger}$
}

Birgit Watzke, Heinz Rüddel, Ralph Jürgensen, Uwe Koch, Levente Kriston, Barbara Grothgar and Holger Schulz

\section{Background}

Although cognitive-behavioural therapy (CBT) and psychodynamic therapy (PDT) are both effective treatments for mental disorders, they show clear dissimilarities concerning their therapeutic models and treatment rationales.

\section{Aims \\ To determine the effectiveness of systematic treatment selection (STS) to CBT or PDT in a mental healthcare setting compared with a control procedure of random treatment selection (RTS). \\ Method \\ A randomised controlled trial in a consecutive sample of 291 in-patients with at least one ICD-10 mental disorder was performed. The primary outcome was symptom severity (General Severity Index of the Symptom \\ Checklist-14) at 6-month follow-up. Health-related quality of}

life was the secondary outcome, determined using the Short Form-8.

\section{Results}

Analyses revealed no general effect for systematic treatment selection. However, there was a differential effect: systematic selection resulted in a better longer-term outcome for PDT, but not for CBT; STS-PDT patients showed a significantly larger reduction in symptom severity than RTS-PDT patients. This difference was not observed in CBT.

\section{Conclusions}

Since systematic treatment selection seems to be able to optimise treatment outcome, at least for PDT, pursuing systematic treatment assignment strategies in mental healthcare settings is a worthwhile endeavour.

\section{Declaration of interest}

None.
Psychotherapy is effective and efficient for treating patients with a variety of mental disorders. ${ }^{1-3}$ If psychotherapeutic treatment (alone or in combination with psychopharmacotherapy) is indicated, the primary task of the psychiatrist or psychotherapist at the initiation of treatment is to offer the appropriate treatment option and to decide which therapeutic approach would be the most beneficial. ${ }^{4}$ This prognostic assessment of the expected outcome of particular treatment methods is part of the daily clinical routine and is an important issue of mental healthcare. The question of 'what works for whom?' and of an adequate and evidence-based treatment selection was identified early as a vital issue of psychotherapy research. ${ }^{5}$ It remains of continuous interest for both practitioners and the scientific community. ${ }^{6,7}$ Treatment selection is salient within healthcare policy, where the current controversial (and sometimes ideologically guided) discussion focuses mainly on diagnostic groups/mental health problems as the only criterion for treatment selection. ${ }^{8}$

For decisions on treatment selection, cognitive-behavioural therapy (CBT) and psychodynamic therapy (PDT) take a key position owing to their widespread application. ${ }^{9}$ There are numerous studies demonstrating the efficacy and effectiveness of both CBT (e.g. Emmelkamp ${ }^{10}$ and Hollon \& Beck ${ }^{11}$ ) and, to a lesser extent, PDT. ${ }^{12}$ Psychodynamic therapy and CBT are clearly distinct from one another, not only with regard to their basic concepts, training modalities and treatment rationales, but also concerning their actual therapeutic implementation (i.e.

†See editorial pp. 83-85, this issue. therapeutic interventions, attitude, style and content). ${ }^{13,14}$ Despite all efforts to develop integrative and adaptive treatment models integrating or combining both methods (e.g. Keller et al ${ }^{15-17}$ ) these are rarely implemented in routine mental healthcare. ${ }^{9}$

Despite the wide application of both CBT and PDT and their distinct procedural differences, to date no empirical research has addressed the question of whether treatment outcomes can be optimised by employing the method of systematic treatment selection $^{7,18}$ to either treatment method by increasing the match between the individual patient and the applied treatment. Some approaches try to improve this match by adapting the applied treatment to the patient's characteristics within one therapeutic concept. However, at least for CBT, results of a randomised controlled trial (RCT) show that individualisation of treatment allowing therapists to use interventions based on their own inclinations did not produce superior effects compared with standardised interventions. ${ }^{19}$

We present results from an RCT under clinically representative conditions comparing systematic treatment selection and random treatment selection for both CBT and PDT in terms of long-term treatment response. Besides the general effectiveness of systematic treatment selection, we also investigated its differential effectiveness for CBT and PDT (i.e. which therapeutic approach benefited the most from systematic treatment selection). We hypothesised that systematic treatment selection is generally superior to random treatment selection because of the expected optimisation of fit between the individual and the selected treatment approach. Concerning differential effectiveness, we hypothesised that PDT benefits more from systematic treatment 
selection than CBT, as patients receiving PDT are considered to require more abilities (e.g. sufficient degree of psychological mindedness, good quality of object relations ${ }^{20}$ ) than those receiving CBT.

We conducted the RCT under clinically representative conditions in order to provide for generalisability in routine practice. We chose an in-patient setting to ensure better monitoring of assignment and treatment.

\section{Method}

Between October 2002 and May 2003, we studied a consecutive sample of in-patients recruited from a psychotherapeutic in-patient unit in Germany. All patients fulfilled the criteria for at least one mental disorder according to ICD-10 (Chapter V). ${ }^{21}$ Patients without a complete diagnostic procedure (i.e. with less than 1 week of in-patient stay) and patients admitted a second time to this specific clinic for treatment continuation (i.e. their treatment was already established prior to admission) were excluded from the study. Additionally, patients meeting specifically defined criteria (extreme under- or overweight with a body mass index $<12$ or $>50 \mathrm{~kg} / \mathrm{m}^{2}$, decompensated tinnitus with the patient's focus being symptomatic treatment, and psychosis-like states) were also excluded from the study, because they would always need to receive systematic treatment selection ('compelled STS') according to the clinic's standards and therefore could not undergo randomisation for clinical and ethical reasons. Because of the naturalistic context of the study, no further exclusion criteria were set.

The unit in which the study took place is part of routine mental healthcare in Germany, which yields larger in-patient psychotherapeutic capacities than healthcare systems of most European countries. There are about 300 000-400 000 in-patient treatments per year in Germany compared with approximately 280000 out-patient treatments. ${ }^{22}$ In contrast to other countries, in-patient psychotherapeutic treatment is very common and an important part of routine care in Germany. The in-patient system focused on in this study serves individuals with a broad spectrum of F-diagnoses based on ICD-10 (main diagnostic groups: depressive, adjustment and anxiety disorders). Hence, the in-patient system in Germany is not directly comparable to in-patient treatment in other countries. However, with regard to the clinical characteristics of the patients (diagnoses, symptom severity), the treatment dose, and the treatment methods delivered, it is rather comparable to the out-patient sector in other countries.

\section{Study design}

The study was approved by the human ethics committee of the responsible medical association. After a complete description of the study to the patients, written informed consent was obtained. Sociodemographic and clinical characteristics were assessed using clinical interviews and self-ratings as well as psychometric testing. This diagnostic procedure was carried out by experienced clinicians. Participants were randomly assigned to either the experimental group (systematic treatment selection, STS) or the control group (random treatment selection, RTS) in a 1:2 ratio. In the experimental group, patients were assigned to either CBT or PDT in a 3:2 ratio using systematic treatment selection (see below). Patients in the control group were randomly assigned to either CBT or PDT in a 3:2 ratio using random treatment selection. The ratio was determined by the capacities of the PDT and CBT treatment facilities in the unit.

We implemented a single-blind randomised trial with two levels of randomisation. The randomisation to systematic and random treatment selection (first randomisation) and the randomisation to CBT or PDT for patients in the control group (second randomisation) were conducted at the University Medical Centre Hamburg-Eppendorf using an external computerised randomisation schedule. According to this procedure, first the study group (STS $v$. RTS) was set by randomisation. Then the treatment method (CBT or PDT) was determined for each patient. In the experimental group (STS), the treatment method was selected according to the decision of the therapeutic team. In the control group (RTS), treatment was selected according to the second randomisation. This information on treatment method (but not the information on belonging to the experimental or the control group) was given to the person in the clinic entering data in the patient file. The patient file included no information on the selection process itself and the assigning therapeutic team was not involved in the further treatment of a patient. Thus, neither therapists nor patients knew which patients belonged to the experimental group and which patients belonged to the control group.

In order to assess the long-term outcome, patients filled in self-rated questionnaires at the beginning of treatment and at follow-up 6 months after termination of treatment. (An additional assessment took place at the termination of treatment; analyses regarding this assessment are not presented here.) The treatment success for systematically and randomly assigned patients at follow-up was compared across both therapeutic methods (general effectiveness of systematic treatment selection) and differentially for CBT and PDT (differential effectiveness).

\section{Systematic treatment selection}

Evidence-based medicine refers to the integration of the best available evidence from systematic research with the individual clinical expertise of the therapist and the preferences of the patient in order to make a decision in medical care. ${ }^{23}$ Applying these principles of evidence-based medicine, systematic treatment selection for either CBT or PDT comprised an expert consensusbased procedure owing to the lack of systematic empirical knowledge. $^{7}$ Systematic treatment selection was determined during the admission phase within a multistage diagnostic procedure integrating clinical interviews and psychometric tests. It was carried out by a multiprofessional team (psychiatrists and psychologists from both treatment approaches) and was based on a consensus decision. The implemented systematic selection was the result of a long-term development within the unit (since the 1990s). The main criteria for STS related to the patient's therapeutic goals (for $86 \%$ of all decisions) and their diagnoses (74\%), with $68 \%$ of all decisions being based on the combination of both criteria. In accordance with, for example, Ambuehl et al ${ }^{24}$ those therapeutic goals focusing on 'the integration of unconscious aspects of experience' and 'the ability to fully allow the experience of emotions' supported a decision for PDT, whereas goals emphasising 'learning to cope with problematic situations effectively' and 'modifying or changing problematic behaviour' facilitated a decision for CBT. In combination with this, patients with an anxiety disorder, eating disorder or a post-traumatic stress disorder, as well as patients lacking a sufficient level of cognitive reflection, received more CBT. Figure 1 displays all criteria and their relevance for systematic treatment selection.

\section{Treatment and treatment integrity}

Both PDT (focal approach) ${ }^{25}$ and $\mathrm{CBT}^{26}$ comprised brief group therapy held in separate departments of the clinic, and were of similar dose and duration (three to four sessions per week with 


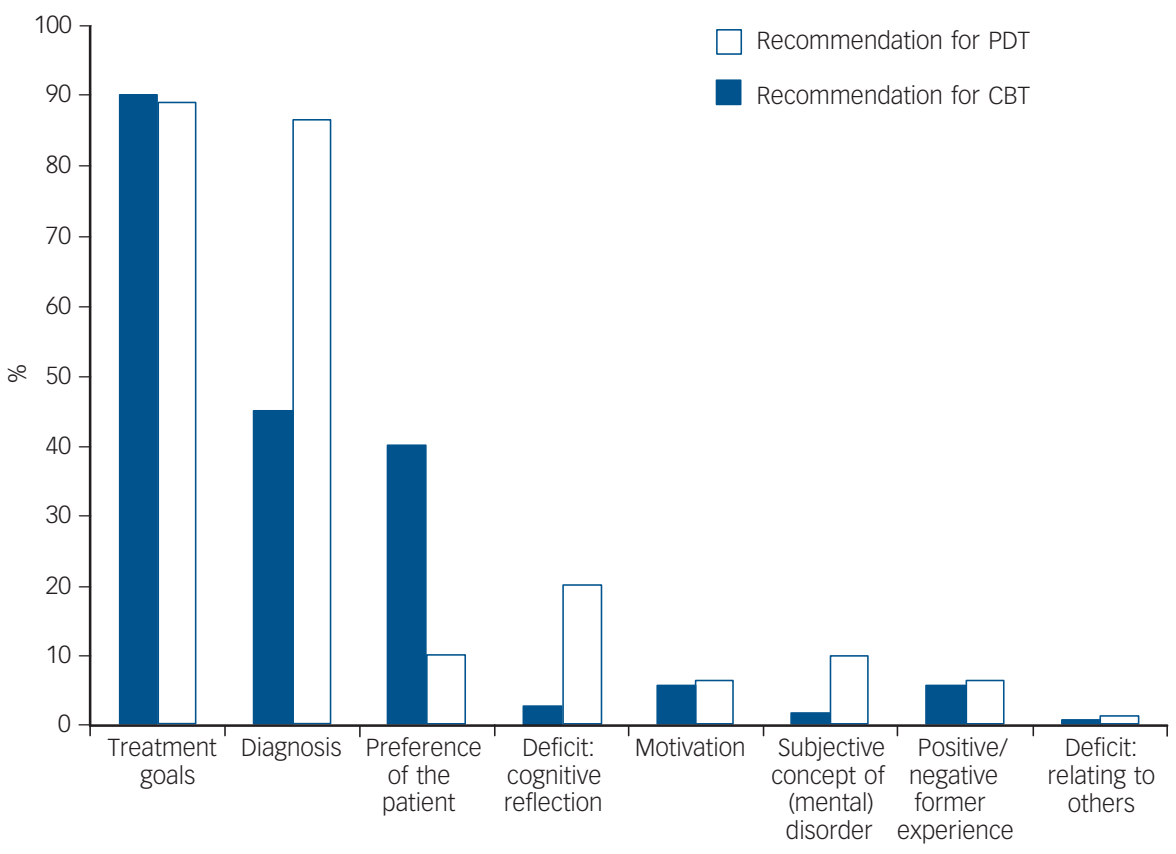

Fig. 1 Criteria for systematic treatment selection (STS) to cognitive-behavioural therapy (CBT) or psychodynamic therapy (PDT) and their relevance for STS (percentage of decisions for which the criteria were referred to).

average treatment duration of 6 weeks). An additional individual short session per week served as an option for the patient to discuss more general therapeutic issues regarding the management of therapy. Patients in both treatment groups (i.e. CBT and PDT) received these sessions.

Because treatment was carried out in routine care, the treatments were not manualised. However, the degree of sufficient treatment differentiation (i.e. adherence to treatment integrity) ${ }^{27}$ between the two therapies was assessed at the unit using videobased expert ratings as well as patient and therapist ratings (patient and therapist checklists were filled out after each session). This assessment of treatment integrity was implemented some months before this trial of STS (i.e. not the same sample of patients but the same therapists as in the present study were investigated). Results confirmed distinct profiles for PDT and CBT according to their therapeutic models: CBT therapists used more cognitive, behavioural and psychoeducational strategies, fostered self-efficacy to a larger extent and were more supporting and empathic. On the contrary, PDT therapists used more interpretive and confrontational interventions concerning intrapsychic issues and focused on interactional aspects as well as on transference and on the past more intensively. ${ }^{14}$

In addition to psychotherapy, about a quarter of the patients took psychotropic medication on a daily basis.

\section{Therapists}

All therapists in the PDT and CBT departments had completed or were of advanced standing in a multiyear postgraduate professional training programme within their specific therapeutic orientation. Psychotherapeutic training in Germany consists of a combination of theoretical education and practical work $(600 \mathrm{~h}$ of theory; $1800 \mathrm{~h}$ of practical work under supervision). After their university degree in psychology or medicine, therapists are trained in either psychodynamic (focal analytic or psychoanalytic) or cognitive-behavioural treatment at specialised, licensed institutions. They then finish their training with an 'Approbation' (licence to practise psychotherapy) for either PDT or CBT. In the unit, all therapists receive supervision from a psychotherapist of the respective therapeutic orientation at least every 2 weeks. Every therapist delivered only one form of treatment.

\section{Outcome measures}

The primary outcome was symptom severity at 6-month followup $\left(T_{1}\right)$ adjusted for symptom severity at baseline $\left(T_{0}\right)$. Symptom severity was assessed using the General Severity Index (GSI) of the Symptom Checklist-14 (SCL-14), a short version of the Symptom Checklist-90-Revised (SCL-90-R), ${ }^{28}$ which comprises nine subscales assessing the current status of symptoms and a summary scale of overall symptom severity (GSI). It is an internationally well-established instrument with proven psychometric properties. $^{28}$ Its internal consistency (Cronbach's alpha) coefficients range from 0.77 to 0.90 and test-retest coefficients from 0.78 to 0.90 . Several studies have demonstrated its concurrent and construct validity as a global measure of symptom severity. The SCL-14 was empirically constructed and refers to three major subscales (depression, anxiety and somatisation) using the original item wording and response format of the SCL-90-R. The GSI of the SCL-14 correlates to $r=0.96$ with the GSI of the SCL-90-R. ${ }^{29}$ Scores vary from 0 ('not at all') to 4 ('extremely'), with higher scores reflecting higher symptom severity.

The secondary outcome was the mental component summary of the Short Form-8 (SF-8, a short version of the SF-36) ${ }^{30}$ assessing health-related quality of life (HRQoL), namely, functional health. Health-related quality of life refers to the degree to which medical problems and disorders, as well as their treatment, impair somatic, emotional and social well-being. This is a superior concept incorporating a variety of dimensions, for example somatic problems, emotional problems, functional impairment, impairments in activity and participation with others. The SF-8 comprises a psychometrically based physical and mental health component summary (PCS and MCS respectively) measure. After standardised norm-based scoring (including recoding and weighting), the resulting summary scores 
vary from 0 ('low HRQoL'/'high impairment in HRQoL') to 100 ('high HRQoL'/'low impairment in HRQoL'), with all scores above and below 50 being above and below the average respectively. In our analyses, we linearly transformed the original scale into a scale ranging from 0 ('no impairment in $\mathrm{HRQoL}$ ') to 4 ('high impairment'), with a score of 2 indicating the average. The instrument is widely used internationally and shows good psychometric properties, with internal consistency from 0.70 to 0.88 and a convergent and discriminant validity shown in several studies. $^{30}$

\section{Statistical analysis}

For the baseline comparison of the experimental (STS) and control (RTS) groups as well as for the comparison of the PDT and CBT patients (in both groups), we analysed demographic and clinical characteristics at $T_{0}$ using $t$-tests for continuous variables and $\chi^{2}$-tests for categorical variables.

To test our hypotheses, both the experimental and control groups were compared using analyses of covariance (ANCOVAs) of $T_{1}$ for the factors assignment (STS-RTS) and treatment method (PDT-CBT) with $T_{0}$ as covariate in order to control for baseline differences. The general effectiveness of systematic selection was assessed with the statistical main effect of the factor assignment. The differential effectiveness of systematic selection for CBT and PDT was assessed with the statistical interaction of assignment $x$ treatment method.

To examine the degree of difference between systematic and random treatment selection, we calculated effect sizes ${ }^{31}$ using partial $\eta^{2}$ for ANCOVAs. Owing to the lack of existing data for the classification of effect sizes, we used Cohen's ${ }^{31}$ interpretation for small (partial $\left.\eta^{2}>0.0099\right)$, medium (partial $\eta^{2}>0.0588$ ) and large (partial $\eta^{2}>0.1379$ ) effect sizes. Partial $\eta^{2}$ can be transformed into other effect sizes, for example into Cohen's $d$ (partial $\eta^{2}=0.0099$ corresponds to $d=0.20$; partial $\eta^{2}=0.0588$ corresponds to $d=0.50$; partial $\eta^{2}=0.1379$ corresponds to $d=0.80)$.

Our primary analyses refer to all patients with complete datasets, i.e. with available assessments at $T_{0}$ and $T_{1}$ (completer analyses). Additionally, we conducted intention-to-treat (ITT) analyses as sensitivity analyses. For ITT analyses, missing values were imputed by carrying forward the baseline value (assuming that individuals who dropped out did not respond to the treatment at all). Therefore we could include all patients with a measurement at baseline in these analyses.

We also conducted drop-out analyses comparing the demographic and clinical baseline characteristics of completers (i.e. primary analyses sample) with non-completers ( $t$ - and $\chi^{2}$-tests).

In order to estimate the possible risk of a selection bias with regard to the recruitment process, analyses of representativeness were additionally carried out on the demographic and clinical baseline characteristics of completers and all other patients being treated in the clinic during the study period and not meeting the exclusion criteria (i.e. patients refusing to participate in the study and non-completers). For the comparison of these two groups we used $t$ - and $\chi^{2}$-tests.

\section{Sample size}

The sample size was determined to provide the study with a statistical power of $80 \%$ for the detection of a small effect between random and systematic treatment selection using ANCOVA, with treatment method as the additional factor and baseline severity as the covariate. Since this is the first study investigating this research question, it seemed appropriate to apply a small effect size. We defined a small effect as $\eta^{2}=0.02$ (corresponding to $f=0.14$ according to Cohen). We assumed that the error variance decreases to an extent of $30 \%$ owing to inclusion of baseline severity as covariate (while being non-correlated to condition assignment owing to randomisation and being moderately correlated to treatment method owing to systematic selection in one of the groups), which led to an adjusted effect size of $\eta^{2}=0.03(f=0.17)$. Setting the level of significance at $\alpha=0.05$, a total sample size of 261 patients was needed. However, because of higher drop-out rates than expected, only 226 patients could be included in the primary analysis (see Results). Thus, the actual power of our analyses was about $74 \%$. In the ITT analyses, the higher number of patients is expected to be compensated by a potential dilution of treatment effects, so the statistical power should be about the same.

\section{Results}

\section{Participants}

Patient flow through the study is shown in the CONSORT diagram in Fig. $2 .{ }^{32}$ Of the 614 patients admitted to the clinic during the study period, 44 were excluded because they were admitted for treatment continuation $(n=20)$ or stayed less than 1 week $(n=24)$. Additionally, $n=65$ patients with 'compelled STS' were excluded from the study because random allocation was not possible for clinical and ethical reasons (as described in the Method). Of the remaining 505 patients, 291 patients (58\%) agreed to participate in the study. The main reasons for study refusal were that patients did not want to be randomised, or were not willing to fill in forms at baseline and at follow-up. A further common reason was that they did not want to take part in research projects in general. These reasons have to be put into perspective - the RCT took place in a clinically representative setting where the conducting of research and the implementation of trials is not very common.

A high response rate at 6-month follow-up (77.7\%) was reached by contacting the patients up to four times (one reminder letter and up to three reminders by telephone). Drop-out rates did not vary substantially between the four study conditions (RTSPDT: 76.3\% completers; RTS-CBT: 78.8\%; STS-PDT: 79.6\%; STS-CBT: $\left.75.9 \% ; \chi^{2}=0.36, P=0.95\right)$. We included 226 patients with complete data-sets in the completer analyses referring to the primary outcome, i.e. the GSI (for the MCS: $n=233$ ).

Baseline clinical and demographic characteristics of the experimental (STS) and control (RTS) groups for the completer and ITT analyses are displayed in Table 1. Despite randomisation, the statistical comparison between the two groups showed a small but systematic difference in GSI and MCS scores, with slightly higher values for patients in the STS groups at baseline (completer analyses: GSI $t(224)=2.0, \quad P=0.045$ and MCS $t(220)=1.9$, $P=0.061$; ITT: GSI $t(275)=2.2, P=0.028$ and MCS $t(265)=2.5$; $P=0.014)$. For all other variables there were no systematic differences between study conditions.

Baseline clinical and demographic characteristics of CBT and PDT patients in both groups are displayed in Table 2. As expected, PDT and CBT patients did not differ significantly in the control group at $T_{0}$. In the experimental group, systematic differences between PDT and CBT were expected as such differences are supposed to be an inherent part of the intervention under investigation (i.e. the systematic treatment selection) owing to the association of certain baseline characteristics with certain factors guiding the treatment selection. In fact, PDT and CBT patients showed clinically relevant differences: PDT patients had a higher professional degree (with $20 \%$ more academics receiving PDT than CBT) and less impairment in HRQoL concerning 


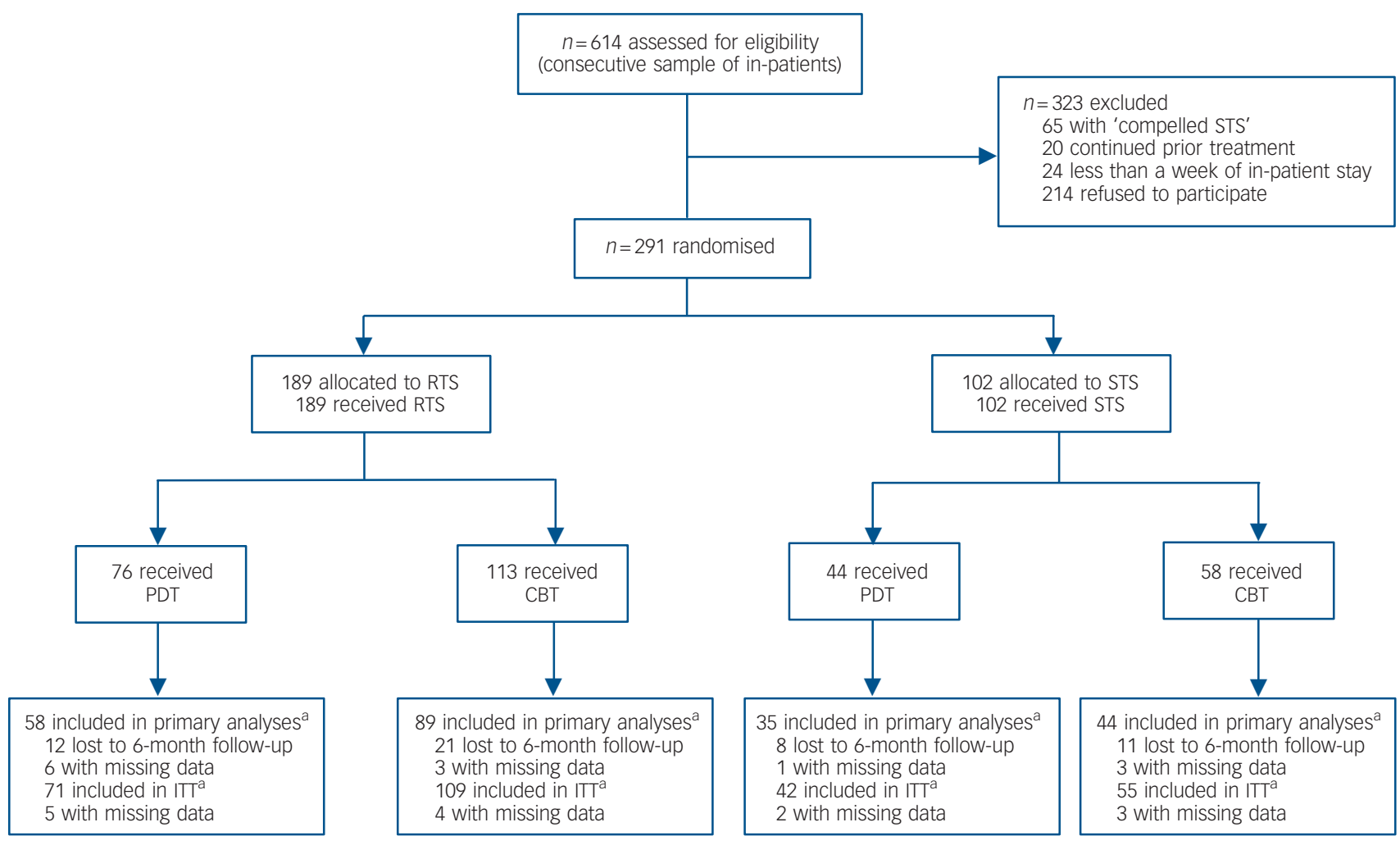

Completer analyses: 147 with RTS; IT: 180 with RTS

Completer analyses: 79 with STS; ITT: 97 with STS

\section{Fig. 2 CONSORT flow of patients through the trial.}

RTS, random treatment selection; STS, systematic treatment selection; PDT, psychodynamic therapy; CBT, cognitive-behavioural therapy. ITT, intention-to-treat analyses. Patients with 'compelled STS', patients with defined criteria who had to receive STS for clinical and ethical reasons (see Method). a. Referring to the analyses of the primary outcome (Global Severity Index of the 14-item Symptom Checklist).

physical aspects (initial SF-8 PCS) than CBT patients allocated to systematic selection. Additionally, there were more women receiving PDT in the STS group ( $80 \% v .68 \%$ receiving CBT).

Psychotropic medication did not differ between CBT and PDT patients in either group (either at $T_{0}$ or at $T_{1}$ ).

The drop-out analyses (i.e. comparison of completers with non-completers) of baseline characteristics revealed a systematic difference regarding age (non-completers were on average 4 years younger; $t(288)=2.1, P=0.039)$ and professional degree (non-completers more often had no professional degree or had not finished it yet; $\left.\chi^{2}=7.81, P=0.05\right)$. Women were slightly overrepresented among completers (completers, $74 \% v$. noncompleters, 63\%). For all other variables being examined (marital status, work incapacity, diagnoses, comorbidity, chronicity, initial scores of SCL-14 and SF-8), no systematic differences between the two groups were found.

The analyses of representativeness (i.e. comparison of completers and all other patients being treated in the clinic during the study period and not meeting the exclusion criteria) also revealed a small systematic difference regarding age (completers were on average 2 years older; $t(502)=2.4$, $P=0.015$ ). For all other variables being examined (gender, marital status, professional degree, work incapacity, diagnoses, comorbidity, chronicity, initial scores of SF-8 and SCL-14), no systematic differences between the two groups were found.

\section{Effectiveness of systematic treatment selection}

Primary analyses (completer analyses)

General effectiveness. The results of the ANCOVAs are displayed in Table 3 and Fig. 3. There was no significant main effect for the factor assignment either for the GSI (STS: marginal mean

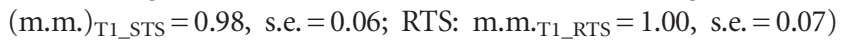
or for the MCS (STS: m.m.T1_STS $=1.81$, s.e. $=0.11$; RTS: $\mathrm{m} . \mathrm{m} \cdot$.T1_RTS $_{1}=1.77$, s.e. $=0.08$ ). Thus, systematically assigned patients did not benefit more from treatment than patients randomly assigned (GSI: $F(1,226)=0.13, P=0.721$, partial $\eta^{2}=0.001 ; \operatorname{MCS}: F(1,233)=0.12, P=0.732$, partial $\left.\eta^{2}=0.001\right)$. This means that, irrespective of treatment method and adjustment for baseline differences, we found no evidence for the general effectiveness of systematic treatment selection.

Differential effectiveness. In assessing the effectiveness of systematic treatment differentially for both CBT and PDT, the ANCOVAs revealed a significant assignment $\times$ treatment method interaction for the primary outcome (GSI: $F(1,226)=4.72$, $P=0.031$, partial $\eta^{2}=0.021$ ). Regarding symptom severity, systematically assigned PDT patients benefited more from the treatment than patients randomly assigned to PDT, although this expected difference was not observed for CBT (Table 3 and Fig. 3). However, we did not find this pattern of differential effectiveness for the secondary outcome, HRQoL. In the ANCOVA, there was 


\begin{tabular}{|c|c|c|c|c|c|c|}
\hline \multirow[b]{2}{*}{ Characteristics } & \multicolumn{3}{|c|}{ Completer analyses } & \multicolumn{3}{|c|}{ Intention-to-treat analyses } \\
\hline & $\begin{array}{c}\text { STS } \\
(n=79)\end{array}$ & $\begin{array}{c}\text { RTS } \\
(n=147)\end{array}$ & $\begin{array}{c}\text { Total } \\
(n=226)\end{array}$ & $\begin{array}{c}\text { STS } \\
(n=97)\end{array}$ & $\begin{array}{c}\text { RTS } \\
(n=180)\end{array}$ & $\begin{array}{c}\text { Total } \\
(n=277)\end{array}$ \\
\hline Female, \% & 73.4 & 74.8 & 74.3 & 72.2 & 73.3 & 72.9 \\
\hline Age, years: mean (s.d.) & $44.5(9.3)$ & $43.4(10.8)$ & $43.8(10.3)$ & $43.4(10.2)$ & 42.8 (110.9) & $43.0(10.7)$ \\
\hline \multicolumn{7}{|l|}{ Professional degree, \% } \\
\hline University degree & 7.1 & 8.8 & 8.2 & 5.7 & 8.5 & 7.5 \\
\hline Non-university degree & 71.4 & 74.5 & 73.4 & 70.5 & 71.5 & 71.1 \\
\hline Without professional degree & 10.0 & 8.0 & 8.7 & 11.3 & 9.7 & 10.3 \\
\hline Other & 11.4 & 8.8 & 9.7 & 12.5 & 10.3 & 11.1 \\
\hline \multicolumn{7}{|l|}{ Work incapacity before treatment, months: \% } \\
\hline$<3$ & 45.9 & 49.1 & 48.0 & 45.5 & 49.3 & 47.9 \\
\hline $3-6$ & 18.0 & 16.7 & 17.1 & 16.9 & 16.4 & 16.6 \\
\hline$>6$ & 19.7 & 15.8 & 17.1 & 19.5 & 14.2 & 16.1 \\
\hline Not employed & 16.4 & 18.4 & 17.7 & 18.2 & 20.1 & 19.4 \\
\hline Number of diagnoses (ICD-10), mean (s.d.) & $3.4(1.4)$ & $3.4(1.4)$ & $3.4(1.4)$ & $3.4(1.4)$ & $3.4(1.4)$ & $3.4(1.4)$ \\
\hline Duration of treatment, days: mean (s.d.) & $42.7(10.3)$ & $42.2(11.5)$ & $42.4(11.1)$ & $41.6(12.0)$ & $41.4(13.3)$ & $41.5(12.8)$ \\
\hline \multicolumn{7}{|l|}{ Diagnoses (ICD-10), ${ }^{\mathrm{b}} \%$} \\
\hline Depression (F32-34) & 40.8 & 42.2 & 41.7 & 42.6 & 41.7 & 42.0 \\
\hline Anxiety disorder (F40, F41) & 17.1 & 12.2 & 13.9 & 16.0 & 11.1 & 12.7 \\
\hline Adjustment disorder (F43.2) & 19.7 & 25.2 & 23.3 & 18.1 & 24.4 & 22.3 \\
\hline PTSD (43.1) & 2.6 & 3.4 & 3.1 & 2.1 & 3.3 & 2.9 \\
\hline Somatoform disorder (F45) & 6.6 & 4.8 & 5.4 & 7.4 & 6.1 & 6.6 \\
\hline Eating disorder (F50) & 9.2 & 4.8 & 6.3 & 8.5 & 5.6 & 6.5 \\
\hline Personality disorder (F60-61) & 0.0 & 0.7 & 0.4 & 2.1 & 1.1 & 1.5 \\
\hline Other mental disorder & 2.6 & 5.4 & 4.5 & 2.1 & 5.6 & 4.4 \\
\hline Somatic diagnosis & 1.3 & 1.4 & 1.3 & 1.1 & 1.1 & 1.1 \\
\hline SF-8 MCS score, mean (s.d.) & $2.52(0.77)$ & $2.30(0.84)$ & $2.37(0.82)$ & $2.56(0.77)$ & $2.30(0.84)$ & $2.39(0.82)$ \\
\hline SF-8 PCS score, mean (s.d.) & $2.18(0.92)$ & $2.22(0.89)$ & $2.20(0.90)$ & $2.20(0.89)$ & $2.22(0.87)$ & $2.21(0.88)$ \\
\hline GSI of the SCL-14 score, mean (s.d.) & $1.39(0.84)$ & $1.17(0.76)$ & $1.25(0.79)$ & $1.40(0.80)$ & $1.18(0.77)$ & $1.26(0.79)$ \\
\hline
\end{tabular}

no significant assignment $\times$ treatment method interaction for this outcome parameter (MCS: $F(1,233)=2.06, P=0.153$, partial $\left.\eta^{2}=0.009\right)$.

\section{Sensitivity analyses (ITT analyses)}

General effectiveness. The results of the ITT analyses (Table 4) confirmed the results of the completer analyses. We found no significant main effect for the factor assignment either for the GSI (STS: m.m.T1_STS $=1.06$, s.e. $=0.06 ;$ RTS: $\mathrm{m} . \mathrm{m} \cdot{ }_{\mathrm{T} 1 \_\mathrm{RTS}}=1.04$, s.e. $=0.05$ ) or for the MCS (STS: $\mathrm{m} . \mathrm{m}_{\text {T1 } 1 \text { STS }}=1.93$, s.e. $=0.10$; RTS: $\mathrm{m} . \mathrm{m}_{\text {.T1_RTS }}=1.88$, s.e. $\left.=0.07\right)$. As in the completer sample, systematically assigned patients did not benefit more from treatment than patients randomly assigned (GSI: $F(1,277)=0.12$, $P=0.734$, partial $\eta^{2}<0.001$; MCS: $F(1,278)=0.17, P=0.679$, partial $\eta^{2}=0.001$ ).

Differential effectiveness. As in the primary analyses, the ANCOVAs for the sample revealed a significant assignment $x$ treatment method interaction for the primary outcome (GSI: $F(1,226)=4.72, P=0.031$, partial $\eta^{2}=0.021$; Table 4) indicating the differential effectiveness of systematic treatment selection regarding symptom severity. For HRQoL, our secondary outcome, the assignment $\times$ treatment method interaction reached no significance, replicating the result of the primary analyses (MCS: $F(1,233)=2.06, P=0.153$, partial $\left.\eta^{2}=0.009\right)$.

\section{Discussion}

We did not find evidence for the general effectiveness of systematic treatment selection in an in-patient sample receiving psychodynamic or cognitive-behavioural therapy. However, the results support the assertion that systematic treatment selection is effective for psychodynamic treatment, but not for the cognitive-behavioural approach. Systematically assigned PDT patients showed a significantly larger reduction in symptom severity 6 months after treatment compared with patients randomly assigned to PDT. For patients treated with CBT, this hypothesised effect was not found.

This result is in line with theoretical considerations about therapy concepts implying that psychodynamic and psychoanalytically oriented therapies require patients with certain abilities (e.g. the ability to reflect or psychological mindedness), ${ }^{20,33,34}$ whereas such explicit considerations do not exist for CBT. Therefore, it might be easier within the cognitivebehavioural model to respond to a broader unselected population presenting for routine care. Problems for patients receiving PDT who do not meet the requirements for this treatment approach may arise at two levels within the therapy process: not only may these patients not respond adequately to PDT interventions, but also difficulties may occur during the actual implementation and delivery of the interventions. As fewer such problems should arise with CBT (because of its wider applicability), possibly resulting differences in treatment delivery could be an alternative interpretation of our study results. 
Table 2 Baseline characteristics of patients assigned to cognitive-behavioural therapy (CBT) and psychodynamic therapy (PDT) in the experimental and control groups (completer analyses) ${ }^{\mathrm{a}}$

\begin{tabular}{|c|c|c|c|c|c|c|}
\hline \multirow[b]{2}{*}{ Characteristics } & \multicolumn{3}{|c|}{ Experimental group (STS) } & \multicolumn{3}{|c|}{ Control group (RTS) } \\
\hline & $\begin{array}{c}\text { CBT } \\
(n=44)\end{array}$ & $\begin{array}{c}\text { PDT } \\
(n=35)\end{array}$ & $t$-test $/ \chi^{2}$ & $\begin{array}{c}\text { CBT } \\
(n=89)\end{array}$ & $\begin{array}{c}\text { PDT } \\
(n=58)\end{array}$ & $\mathrm{t}$-test $/ \chi^{2}$ \\
\hline Age, years: mean (s.d.) & $43.8(8.6)$ & $45.3(10.1)$ & $t(77)=0.72, P=0.47$ & $42.0(10.9)$ & $45.5(10.2)$ & $t(144)=1.92, P=0.056$ \\
\hline Female, \% & 68.2 & 80.0 & $\chi^{2}(1, n=79)=1.39, P=0.23$ & 74.2 & 75.9 & $\chi^{2}(1, n=147)=0.54, P=0.81$ \\
\hline Professional degree, \% & & & & & & \\
\hline University degree & 2.5 & 13.3 & $\chi^{2}(3, n=101)=7.73, P=0.052$ & 10.0 & 7.0 & $\chi^{2}(3, n=137)=4.37, P=0.22$ \\
\hline Non-university degree & 80.0 & 60.0 & & 68.8 & 82.5 & \\
\hline Without professional degree & 12.5 & 6.7 & & 8.8 & 7.0 & \\
\hline Other & 5.0 & 20.0 & & 12.5 & 3.5 & \\
\hline
\end{tabular}

Work incapacity before treatment, months: $\%$

\begin{tabular}{|c|c|c|c|c|c|c|}
\hline$<3$ & 39.4 & 53.6 & $\chi^{2}(3, n=61)=1.29, P=0.73$ & 49.3 & 48.9 & $\chi^{2}(3, n=114)=2.77, P=0.42$ \\
\hline $3-6$ & 21.2 & 14.3 & & 18.8 & 13.3 & \\
\hline$>6$ & 21.2 & 17.9 & & 11.6 & 22.2 & \\
\hline Not employed & 18.2 & 14.3 & & 20.3 & 15.6 & \\
\hline $\begin{array}{l}\text { Jumber of diagnoses (ICD-10), } \\
\text { nean (s.d.) }\end{array}$ & $3.5(1.4)$ & $3.1(1.3)$ & $t(74)=-1.34, P=0.18$ & $3.4(1.4)$ & $3.4(1.2)$ & $t(134)=0.22, P=0.82$ \\
\hline $\begin{array}{l}\text { uration of treatment, days: } \\
\text { nean (s.d.) }\end{array}$ & $44.5(10.7)$ & $40.2(9.3)$ & $t(77)=-1.84, P=0.06$ & $43.2(12.7)$ & $40.5(9.1)$ & $t(143)=-1.50, P=0.13$ \\
\hline F-8 MCS score, mean (s.d.) & $2.46(0.78)$ & $2.59(0.75)$ & $t(75)=0.73, P=0.46$ & $2.32(0.87)$ & $2.25(0.79)$ & $t(143)=-0.45, P=0.65$ \\
\hline F-8 PCS score, mean (s.d.) & $2.32(0.91)$ & $2.00(0.90)$ & $t(76)=-1.54, P=0.12$ & $2.17(0.93)$ & $2.28(0.81)$ & $t(143)=0.70, P=0.48$ \\
\hline CL-14 GSI score, mean (s.d.) & $1.40(0.91)$ & $1.37(0.75)$ & $t(77)=-0.15, P=0.87$ & $1.17(0.80)$ & $1.17(0.69)$ & $t(145)=0.23, P=0.98$ \\
\hline
\end{tabular}

STS, systematic treatment selection; RTS, random treatment selection; SCL-14, Short version of the Symptom Checklist; GSI, Global Severity Index; SF-8 MCS, short version of the SF-36 Health Survey, mental component summary; SF-8 PCS, short version of the SF-36 Health Survey, physical component summary.

a. $n=226$ participants with complete data-sets (completer sample). All $P$ values are two-sided.

b. This refers to a 1 -year period before treatment.

Table 3 Comparison of systematic (STS) and random treatment selection (RTS) at follow-up (completer analyses)

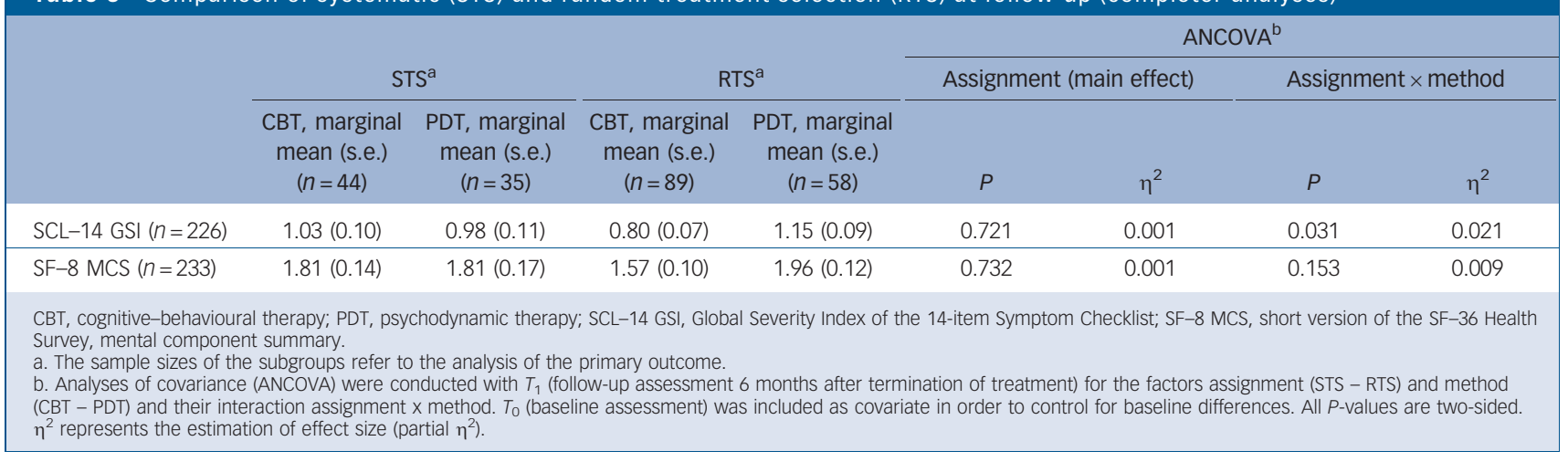

Table 4 Comparison of systematic (STS) and random treatment selection (RTS) at follow-up (intention-to-treat analyses)

\begin{tabular}{|c|c|c|c|c|c|c|c|c|}
\hline & & & & & \multicolumn{4}{|c|}{ ANCOVA $^{\mathrm{b}}$} \\
\hline & \multicolumn{2}{|c|}{ STS $^{\mathrm{a}}$} & \multicolumn{2}{|c|}{ RTS $^{\mathrm{a}}$} & \multicolumn{2}{|c|}{ Assignment (main effect) } & \multicolumn{2}{|c|}{ Assignment $\times$ method } \\
\hline & $\begin{array}{l}\text { CBT, marginal } \\
\text { mean (s.e.) } \\
(n=550)\end{array}$ & $\begin{array}{l}\text { PDT, marginal } \\
\text { mean (s.e.) } \\
(n=42)\end{array}$ & $\begin{array}{c}\text { CBT, marginal } \\
\text { mean (s.e.) } \\
(n=109)\end{array}$ & $\begin{array}{c}\text { PDT, marginal } \\
\text { mean (s.e.) } \\
(n=71)\end{array}$ & $P$ & $\eta^{2}$ & $P$ & $\eta^{2}$ \\
\hline SCL-14 GSI $(n=277)$ & $1.08(0.08)$ & $1.05(0.09)$ & $0.89(0.06)$ & $1.18(0.07)$ & 0.734 & $<0.001$ & 0.044 & 0.015 \\
\hline SF-8 MCS $(n=278)$ & $1.93(0.13)$ & $1.93(0.15)$ & $1.72(0.09)$ & $2.03(0.11)$ & 0.679 & 0.001 & 0.206 & 0.0096 \\
\hline \multicolumn{9}{|c|}{$\begin{array}{l}\text { CBT, cognitive-behavioural therapy; PDT, psychodynamic therapy; SCL-14 GSI, Global Severity Index of the 14-item Symptom Checklist; SF-8 MCS, short version of the SF-36 Healtt } \\
\text { Survey, mental component summary. } \\
\text { a. The sample sizes of the subgroups refer to the analysis of the primary outcome. } \\
\text { b. Analyses of covariance (ANCOVA) were conducted with } T_{1} \text { (follow-up assessment } 6 \text { months after termination of treatment) for the factors assignment (STS - RTS) and method } \\
\text { ('BT - PDT) and their interaction assignment } x \text { method. } T_{0} \text { (baseline assessment) was included as covariate in order to control for baseline differences. All P-values are two-sided. } \\
\eta^{2} \text { represents the estimation of effect size (partial } \eta^{2} \text { ). }\end{array}$} \\
\hline
\end{tabular}




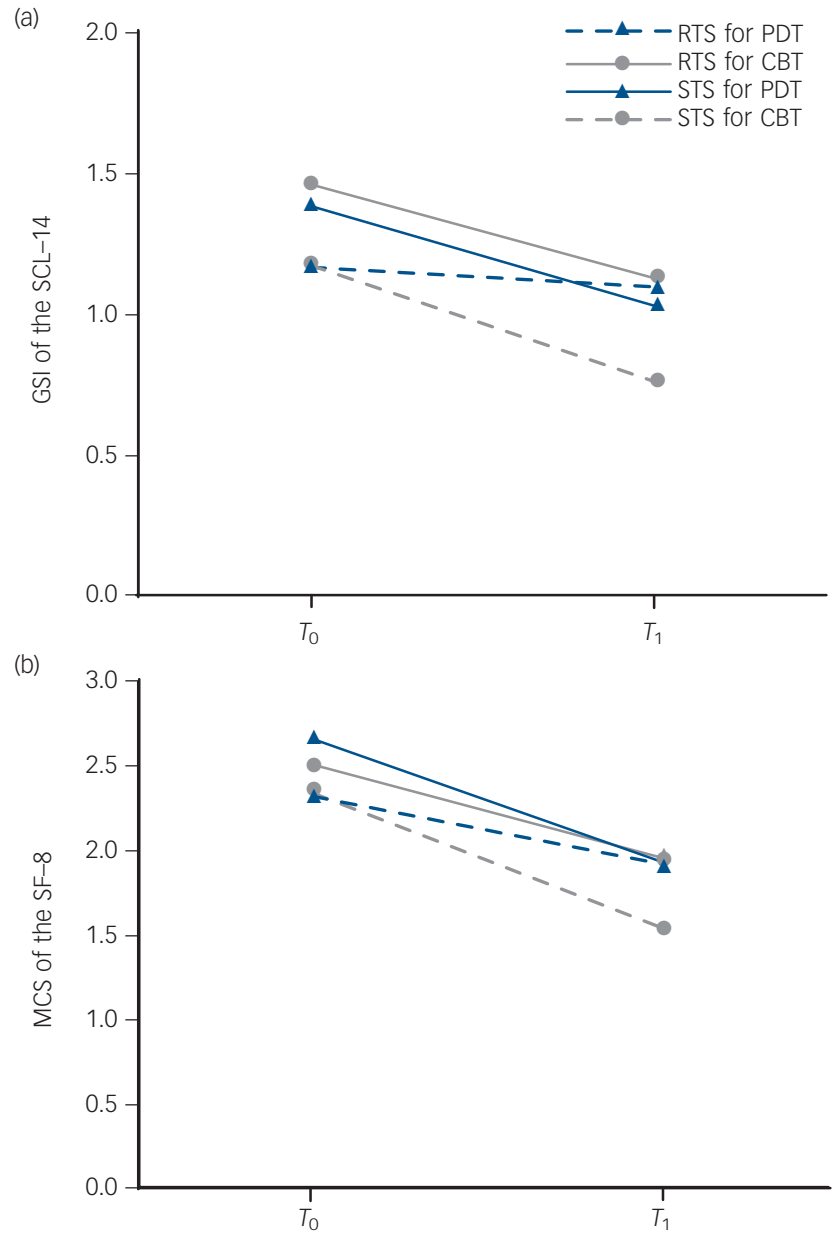

Fig. 3 Improvement from baseline $\left(T_{0}\right)$ to follow-up $\left(T_{1}\right)$ for the study groups (systematic treatment selection, STS $v$. random treatment selection, RTS) for cognitive-behavioural therapy (CBT) and psychodynamic therapy (PDT) patients in terms of (a) symptom severity $(n=226)$ and (b) HRQOL $(n=233)$. For both variables, higher scores (mean values) reflect higher degrees of distress or impairment.

SCL-14 GSI, Global Severity Index of the 14-item Symptom Checklist; SF-8 MCS, short version of the SF-36 Health Survey, mental component summary; $T_{0}$, assessment at the beginning of treatment (baseline); $T_{1}$, follow-up assessment 6 months after the termination of treatment.

The results of a previous study on prognostic assessment of therapy outcome are also consistent with our findings with respect to PDT: Spinhoven et al ${ }^{35}$ found that assessors' ratings at baseline significantly predicted which patients with borderline personality disorder failed to change reliably or had not recovered after 3 years of transference-focused psychotherapy, although they could not predict similar outcomes in patients treated with CBT.

According to our data, the effectiveness of systematic treatment selection for the cognitive-behavioural approaches can neither be confirmed nor ruled out. The treatment improvement seen in randomly assigned CBT patients at least equals that of systematically assigned patients. Considering this data pattern, along with the result that systematically assigned CBT patients seem to have more disadvantageous prognostic factors for improvement (lower professional degree indicating lower education, higher impairment in physical HRQoL) than patients in the other study groups, it can be assumed that instead of explicit CBT indications, PDT contraindications were more likely to lead to a CBT assignment in our study. This would mean that in the STS group, patients who have a poorer prognosis for psychotherapeutic treatment in general were assigned to CBT, whereas in the RTS group, patients were evenly distributed among both treatment methods. This might be also a reason for the inability to detect a general effect of systematic treatment selection (v. random treatment selection) in our study.

One further interesting result refers to the finding that differential effectiveness showed a significant effect for symptom severity, but not for our secondary outcome (HRQoL). However, the resulting slopes for HRQoL showed at least a similar pattern for the four study groups as those for symptom severity.

The sensitivity analyses (ITT analyses), including all patients with a baseline assessment, revealed a significant effect for the differential effectiveness of systematic treatment selection, but not for its general effectiveness. This replicates and confirms the results of the completer analyses, supporting that there was no substantial attrition bias due to drop-out at the follow-up assessment.

The effect of the treatment method itself (CBT/PDT) refers to a further interesting and very relevant research question (regarding the long tradition of outcome research comparing different treatment approaches). However, this is beyond the scope of this paper.

One limitation of the study is the participation rate (58\%), which was lower than expected. However, it must be considered that we recruited a consecutive sample of in-patients (with only a few exclusion criteria) in routine mental healthcare. Additionally, the representativeness analyses, which showed no systematic differences between participants and non-participants, except that of age, contribute to the generalisability of the findings.

A further shortcoming might be seen in the exclusion of the group of patients who had to be systematically randomised according to the clinic's standards (compelled STS). Although for these patients systematic treatment selection seemed to be especially important, the effects could not be evaluated because randomisation to either systematic or random selection was not possible for clinical and ethical reasons.

As we were unable to carry out the relatively expensive procedure of a structured diagnostic interview (e.g. Structured Clinical Interview for DSM Disorders) ${ }^{36}$ at the clinic, the diagnoses of our patient sample are based solely on the clinical judgement of the therapists. Although these were experienced clinical psychotherapists with extensive 3- or 5-year training, including comprehensive modules of diagnostic assessment, there is a certain risk of unreliable diagnoses. ${ }^{37}$ Therefore, although the aim of our study was not to focus on diagnostic subgroups, an additional standardised diagnostic procedure would have been desirable but was not feasible because this study was carried out in routine care.

For the same reason, treatments were not manualised but represent PDT and CBT as conducted in routine care. This implicates certain strengths with regard to external validity, but also leads to uncertainties regarding treatment integrity and treatment differentiation. Our naturalistic study could not reach the gold standard of trials in experimental settings, which refer to manualised treatments and therapists with specialised training. However, the results of our additional trial on treatment integrity (being conducted with the same therapists but not the same patients $)^{14}$ at least support a sufficient degree of treatment integrity in the clinic under investigation.

A further potential shortcoming is the slightly different baseline scores of the experimental (STS) and the control (RTS) group in symptom severity and HRQoL. These small differences 
might have been relevant if there had been significant effects regarding systematic treatment selection in general (for both CBT and PDT), but they are not a plausible alternative explanation for the differential effects (i.e. an effect for PDT, but not for CBT) found in the study. Nevertheless, despite controlling statistically for the differences (ANCOVA) it cannot be absolutely ruled out that the slightly lower initial scores in the control group have led to a bias that overlies the hypothesised superiority of systematic treatment selection. However, the similar gradients of improvement in three of the four study conditions (RTS-CBT; STS-CBT and STS-PDT) do not support this explanation.

Another limitation might be seen in the applied criteria for systematic treatment selection. As the empirical knowledge for criteria and their operationalisations is marginal, we referred to the 'best available evidence' in the sense of evidence-based medicine, ${ }^{23}$ a procedure based on expert consensus. However, we do not know whether this procedure is directly reproducible across other diagnostic and treatment settings.

The study implemented an experimental double-blind design in routine in-patient health services by masking both therapists and patients to the information on study group (STS/RTS). This enabled us to assess the long-term effects of a systematic treatment selection model for psychotherapeutic treatments on symptom severity and HRQoL while ruling out expectancy effects that might play an important role in psychotherapeutic treatment. According to the results, systematic treatment selection seems to be able to optimise treatment outcome, at least for the psychodynamic approaches in routine care. Therefore, pursuing strategies for systematic assignment procedures in mental healthcare settings seems worthwhile. In order to be able to succeed in this and to optimise routine care by means of evidence-based procedures, it is necessary to strengthen research on specific prognostic markers for particular types of treatment. With this empirical knowledge it might be possible to develop feasible criteria for systematic treatment selection which can be reliably and validly assessed with standard procedures in clinical practice.

Birgit Watzke, PhD, Department of Medical Psychology, University Medical Center Hamburg-Eppendorf; Heinz Rüddel, MD, Ralph Jürgensen, St. Franziska-Stift, Bad Kreuznach; Uwe Koch, PhD, MD, Levente Kriston, PhD, Department of Medical Psychology, University Medical Center Hamburg-Eppendorf; Barbara Grothgar, PhD, St. Franziska-Stift, Bad Kreuznach; Holger Schulz, PhD, Department of Medical Psychology, University Medical Center Hamburg-Eppendorf, Germany

Correspondence: Birgit Watzke, Research Group for Mental Health Care Research Department of Medical Psychology, University Medical Center Hamburg-Eppendorf, Martinistraße 52 (Building W26), Hamburg 20246, Germany. Email: watzke@uke.uni-hamburg.de

First received 10 Sep 2010, final revision 5 Jan 2010, accepted 8 Mar 2010

\section{Funding}

Funded by the German Research Foundation (Deutsche Forschungsgemeinschaft, DFG). Grant No. SCHU 1513/1-1 to H.S.

\section{Acknowledgements}

The authors thank Julia Deubel and Martina Kraemer for assisting with the study.

\section{References}

1 Shadish WR, Matt GE, Navarro AM, Phillips G. The effects of psychological therapies under clinically representative conditions - a meta-analysis. Psychol Bull 2000; 126: 512-29.
2 Luty SE, Carter JD, McKenzie JM, Rae AM, Frampton CMA, Mulder RT, et al. Randomised controlled trial of interpersonal psychotherapy and cognitivebehavioural therapy for depression. Br J Psychiatry 2007; 190: 496-502.

3 Lambert MJ, Ogles BM. The efficacy and effectiveness of psychotherapy. In Bergin and Garfield's Handbook of Psychotherapy and Behavior Change (ed MJ Lambert): 139-93. John Wiley, 2003.

4 Tillett R. Psychotherapy assessment and treatment selection. Br J Psychiatry 1996; 168: 10-5.

5 Paul GL. Strategy of outcome research in psychotherapy. J Consult Psychol 1967; 31: 109-18.

6 Roth A, Fonagy P. What Works for Whom? A Critical Review of Psychotherapy Research. Guilford Press, 1996.

7 Beutler LE, Harwood TM. Systematic treatment selection and prescriptive therapy. In A Casebook of Psychotherapy Integration (eds G Stricker, J Gold): 29-41. American Psychological Association, 2006.

8 Chambless DL, Ollendick TH. Empirically supported psychological interventions: Controversies and evidence. Annu Rev Psychol 2001; 52: 685-716.

9 Barghaan D, Schulz H, Koch U, Watzke B. Versorgungsstrukturen im stationären Setting in Deutschland: Verteilung von Einzel- und Gruppentherapie und deren psychotherapeutischen Ausrichtungen [Structure of inpatient mental health care in Germany: distribution of individual and group therapy and their treatment approaches]. Gruppenpsychother Gr 2009; 45: 83-103.

10 Emmelkamp PMG. Behavior therapy with adults. In Bergin and Garfield's Handbook of Psychotherapy and Behavior Change (ed MJ Lambert): 393-446. John Wiley, 2003.

11 Hollon SD, Beck AT. Cognitive and cognitive behavioral therapies. In Bergin and Garfield's Handbook of Psychotherapy and Behavior Change (ed MJ Lambert): 447-92. John Wiley, 2003.

12 Leichsenring F, Rabung S, Leibing E. The efficacy of short-term psychodynamic psychotherapy in specific psychiatric disorders: a metaanalysis. Arch Gen Psychiat 2004; 61: 1208-16.

13 Goldfried MR, Castonguay LG. A comparative analysis of the therapeutic focus in cognitive-behavioral and psychodynamic-interpersonal sessions. J Consult Clin Psychol 1997; 65: 740-8.

14 Watzke B, Rueddel H, Koch U, Rudolph M, Schulz H. Comparison of therapeutic action, style and content in cognitive-behavioural and psychodynamic group therapy under clinically representative conditions. Clin Psychol Psychother 2008; 15: 404-17.

15 Keller MB, McCullough JP, Klein DN, Arnow BA, Rush AJ, Nemeroff CB, et al. A comparison of nefazadone, the cognitive behavioral-analysis system of psychotherapy, and their combination for the treatment of chronic depression. N Engl J Med 2000; 322: 1462-70.

16 Feixas G, Botella L. Psychotherapy Integration: reflections and contributions from a constructivist epistemology. J Psychother Integrat 2004; 14: 192-222.

17 Grawe K. Psychological Therapy. Hogrefe \& Huber Publishers, 2002.

18 Harwood TM, Williams OB. Identifying treatment relevant assessment: the STS. In Integrative Assessment of Adult Personality (eds LE Beutler, G GrothMarnat): 65-81. Guilford Press, 2003.

19 Schulte D, Kunzel R. Tailor-made versus standardized therapy of phobic patients. Adv Behav Res Ther 1992; 14: 67-92.

20 Valbak K. Suitability for psychoanalytic psychotherapy: a review. Acta Psychiatr Scand 2004; 109: 164-78.

21 World Health Organization. The ICD-10 Classification of Mental and Behavioural Disorders. Clinical Descriptions and Diagnostic Guidelines. WHO, 1992.

22 Schulz H, Barghaan D, Harfst T, Koch U. Gesundheitsberichterstattung des Bundes: Psychotherapeutische Versorgung [Federal Health Reporting: Mental Health Care]. Robert-Koch-Institut, 2008

23 Sackett DL, Rosenberg WM. Evidence based medicine: what it is and what it isn't. BMC 1996; 312: 71-2.

24 Ambühl H, Orlinsky DE. Zum Einfluss der theoretischen Orientierung auf die psychotherapeutische Praxis [How does the theoretical orientation influence the psychotherapeutic work?]. Psychotherapeut 1997; 42: 290-8.

25 Heigl- Evers A, Ott J. Die Bedeutung der psychoanalytisch-interaktionellen Grundeinstellungen für die Gruppentherapie [Relevance of psychoanalyticinteractional attitude for group psychotherapy]. Gruppenpsychother Gr 1997; 33: 55-73.

26 Fiedler P. Verhaltenstherapie in und mit Gruppen [Cognitive-behavioural therapy in the group setting]. In Praxis der Gruppenpsychotherapie (ed V Tschuschke): 343-8. Thieme, 2001. 
27 Waltz J, Addis ME, Koerner K, Jacobsen N. Testing the integrity of a psychotherapeutic protocol: Assessment of adherence and competence. J Consult Clin Psych 1993; 61: 620-30.

28 Derogatis LR. SCL-90-R. Administration, Scoring and Procedures Manual-I for the R(evised) Version. John Hopkins University School Medicine, 1977.

29 Harfst T, Koch U, Kurtz von Aschoff C, Nutzinger DO, Rüddel H, Schulz H Entwicklung und Validierung einer Kurzform der Symptom Checklist-90-R [Development and validation of a short evrsion of the symptom checklist-90-R]. DRV-Schriften 2002; 33: 71-3.

30 Ware JE. SF-36 Health survey update. Spine 2000; 25: 3130-9.

31 Cohen J. Statistical Power Analysis for the Behavioral Sciences. Lawrence Erlbaum, 1988.

32 Begg C, Cho M, Eastwood S, Horton R, Moher D, Olkin I, et al. Improving the quality of reporting of randomized controlled trials. The CONSORT statement. JAMA 1996; 276: 637-9.
33 Taylor GJ. Psychoanalysis and empirical research: the example of patients who lack psychological mindedness. J Am Acad Psychoanal 1995; 23: 263-81.

34 Grand S. A classic revisited: clinical and theoretical reflections on Stone's Widening scope of indications for psychoanalysis. J Am Psychoanal Ass 1995; 43: 741-64.

35 Spinhoven P, Giesen-Bloo J. Can assessors and therapists predict the outcome of long-term psychotherapy in borderline personality disorder. J Clin Psychol 2008; 64: 667-86.

36 First MB, Spitzer RL, Gibbon M, Williams JBW. Structured Clinical Interview for DSM-IV Axis I Disorders, Clinician Version (SCID-CV). American Psychiatric Press, 1996.

37 Shear MK, Greeno C, Kang J, Ludewig D, Frank E, Swartz HA, et al. Diagnosis of nonpsychotic patients in community clinics. Am J Psychiatry 2000; 157 . $581-7$.

\section{psychiatry in the Old Testament}

\section{Early child psychiatry}

\section{George Stein}

Disturbed and disobedient children can cause much distress to their parents, sometimes precipitating maternal depression. In the Book of Proverbs the word 'fool' denotes a character corresponding to the modern concept of personality disorder (mainly antisocial). Parental grief or depression associated with having a fool as a child is described in three separate entries:

10:1 'A wise child makes a glad father, but a foolish child is a mother's grief.'

17:21 'The one who begets a fool gets trouble; the parent of the fool has no joy.'

17:25 'Foolish children are a grief to their father, and bitterness to her who bore them.'

In the recent child psychiatry literature numerous studies replicate this association between maternal depression and having children with conduct disorder, thus confirming the views of the scribes of the Book of Proverbs.

But what was the management of the more seriously disturbed and delinquent adolescents, a group that still poses a major challenge to services today? Here the parents liaised with the local 'social services' (the elders) and the solution they came up with is described in Deuteronomy 21:18-21.

21:18 'If someone has a stubborn and rebellious son who will not obey his father and mother, who does not heed them when they discipline him, 19 Then his father and mother shall take hold of him and bring him out to the elders of his town at the gate of that place. 20 They shall say to the elders of his town "This son of ours is stubborn and rebellious. He will not obey us. He is a glutton and a drunkard." 21 Then all the men of the town shall stone him to death. So you shall purge the evil from your midst; and all Israel will be afraid.'

In the earlier patriarchal period of ancient Israel and also in ancient Rome, parents had the absolute right to execute their children. However, under this deuteronomic ruling an execution could only occur following a trial and on the authority of the elders; the killing was conducted by all the men of the town, but not the parents. It has been suggested that this option was rarely, if ever, applied and the law was used to strike fear and deterrence into the minds of the defiant youngsters. Curiously, the punishment for the insubordinate son in ancient Mesopotamia as recorded in the much older Code of Hammurabi (circa 1800 BCE) was rather less severe, involving cutting off the delinquent's hands.

Society still has no answer for the rebellious, drunken, violent adolescent. However, secure adolescent provision and prison for the most dangerous individuals is a clinically useful advance over the biblical solution. 